\title{
692 TIM-3 EXPRESSION DRIVES PHENOTYPIC AND FUNCTIONAL CHANGES IN TREG IN SECONDARY LYMPHOID ORGANS AND THE TUMOR MICROENVIRONMENT, EFFECTING TUMOR BURDEN
}

${ }^{1}$ Hridesh Banerjee*, ${ }^{2}$ Hector Nieves-Rosado, ${ }^{1}$ Benjamin Murter, 'Lawrence Kane. 'University of Pittsburgh, Pittsburgh, PA, United States; ${ }^{2}$ UPMC, Pittsburgh, PA, United States

Background Regulatory $\mathrm{T}$ cells ( $\mathrm{T}$ reg) are critical mediators of self-tolerance but can also limit effective anti-tumor immunity. We and others previously reported that $40-60 \%$ percent of $\mathrm{T}$ reg-infiltrating head and neck cancer (HNC) and other tumors highly express Tim-3, compared with about 5\% in lymphoid organs, it therefore gets imperative to characterize if Tim-3 is driving any $\mathrm{T}$ reg specific function in tumor microenvironment and under homeostasis.

Methods Using a conditional TIM-3 inducible and knockout mouse model developed in our lab, we have performed syngeneic tumor challenges in $\mathrm{T}$ reg-specific Tim-3 transgenic and knockout mice (FoxP3ERT2CreSFS-Tim-3 and FoxP3ERT2CreFLEX4). We have also characterized the tumor immune infiltrate of these mice to understand the impact of $\mathrm{T}$ reg specific Tim-3induction and deficiency on the immune landscape.

Results Tim-3 induction on T reg leads to rapid growth associated with higher progression of CD8 compartment towards exhaustion, while TIm-3 knockout in $\mathrm{T}$ reg specific manner leads top overall decline in $\mathrm{T}$ reg compartment in tumors associated with lower exhaustion in the CD8 compartment and decrease in tumor burden,

Conclusions Tumor-infiltrating Tim-3+ Treg have enhanced suppressive function and display a more effector-like phenotype. Using a novel mouse model with cell type-specific Tim-3 expression, we show here that expression of Tim-3 by Treg is sufficient to drive Treg to a more effector-like phenotype, and increases suppressive activity, effector $\mathrm{T}$ cell exhaustion and tumor growth. We also show that inducible deletion of Tim-3 specifically from Treg enhances anti-tumor immunity and decrease in tumor burden along with a decrease in tumor associated Treg compartment. These findings may help to reconcile previous reports that some Tim-3 antibodies enhance $\mathrm{T}$ cell responses in vivo, while expression of Tim-3 has a cellintrinsic ability to enhance TCR signaling and T cell activation. A major role of Tim-3 was found to be mediated through IL-10 and IL-10 R pathway in both Treg and CD8 compartment. Thus, we propose that Tim-3 regulates antitumor immunity at least in part through enhancement of Treg function. To our knowledge, this is the first example in which expression of a single co-stimulatory molecule is sufficient to drive differentiation of Treg in this manner.

Acknowledgements We acknoledge Dr. Robert L. Ferris and Dr. Greg M. Delgoffe for their inputs and guidance with human and metabolism associated experiments.

http://dx.doi.org/10.1136/jitc-2021-SITC2021.692 